\title{
Serum Uric Acid Levels As an Independent Short Term Prognostic Marker in Acute Myocardial Infarction
}

\author{
Authors \\ Dr Padmakumar Rajasekhara Pillai ${ }^{1}$, Dr Suresh Muthezhathu Kesavadas ${ }^{2}$ \\ ${ }^{1}$ Assistant Professor, Government Medical College, Thiruvanthapuram \\ ${ }^{2}$ Professor, Government Medical College, Thiruvanthapuram
}

\section{Background of study}

Studies have shown that elevated serum uric acid level is highly predictive of mortality and morbidity in patients with coronary heart disease. Uric acid is an indicator for increased oxidative stress. Xanthine oxidase, a critical enzyme in degradation of purines to uric acid has shown to be an important source of superoxide free radical. Activity of xanthine oxidase increases during ischemia, clinically hyperuricemia occurs in hypoxic states. The goal of the study was to assess the prognostic value of serum uric acid in the outcome of acute myocardial infarct, both mortality and morbidity. Xanthine oxidase activity and uric acid synthesis are increased in vivo under ischemia and hence elevated uric acid may act as a marker for ischemia.

\section{Introduction}

The association of serum uric acid with cardiovascular disease has been appreciated for nearly half a centuary. ${ }^{1}$ Several prospective studies have shown an association between baseline hyperuricemia and incident coronary heart disease. The LIFE study ${ }^{4}$-showed that Losartan, Atorvastatin, Fenofibrate risk reduction the rapies, are due to serum uric acid reduction. The LIFE ${ }^{4}$ study observed that $29 \%$ of the reduction in composite end point of death in myocardial infarction, is seen with the use of losartan and this was attributable to a decrease in the serum uric acid; Implying a link of serum uric acid with both stroke and coronary heart disease.

A 12 year longitudinal study in Sweden by Bengtsson ${ }^{2}$ et al showed that uric acid had positive correlation with 12 year mortality in univariate analysis $^{2}$. The association between serum uric acid concentration and mortality was independent of age, body mass index, systolic blood pressure, adipose tissue distribution, smoking habit, serum cholesterol concentration and serum triglyceride concentration. NHANES ${ }^{3}$ found a significantly stronger association between time varying serum uric acid and cardiovascular outcome in women than in men. Using data from the NHANES $1^{3}$ study increment in uric acid by $60 \mathrm{mmol} / \mathrm{l}$ was associated with a $48 \%$ increase in risk for incident ischemic heart disease among women. In men uric acid levels gradually increased with age. Gerber et $\mathrm{al}^{9}$ also documented increased incidence of cardiovascular mortality among hyperuricemic subjects. Maden et al showed uric acid level predicted mortality in angiographic significant individuals. Study in Tel Aviv Souraski medical 
centre by Wasserman et al ${ }^{8}$ showed initial serum uric acid an independent predictor of mortality. Monica Augsburg cohort data from 1044 men showed a strong positive correlation of serum uric acid withall-cause mortality. Michael et al showed a strong positive correlation of uric acid and myocardial infarction in 4385 participants of age $>55$ years. Korean study by Hong Lim et al on 716 patient who underwent coronary angiography showed a strong association with an increased risk of coronary artery disease and serum uric acid in women, than in man. Bano et $\mathrm{al}^{6}$ studied level of uric acid in heart failure according to NYHA functional class; found a strong positive correlation of uric acid and severity of heart failure (class IV-43\%,class III 37\%,class II- 20\%, class I $-13 \%$ class $0-7 \%$ ). Japanese study by Kojima et $\mathrm{al}^{7}$ showed serum uric acid level correlate with Killip classification, and high uric acid was associated with poor prognosis in a study of 1024 patients hospitalised within $48 \mathrm{hrs}$ of chest pain. Serum uricacid reflects increased xanthine oxidase activity related to oxidative stress. Serum uric acid level is a suitable marker for predicting adverse event after acute myocardial infarction

\section{Aim}

- To estimate serum uric level in patients with myocardial infarction within $48 \mathrm{hrs}$ of chest pain.

- To observe for correlation between serum uric level and immediate post MI complications.

- To observe correlation between Serum uric acid level and severity of cardiac failure using Killips class.

- To observe correlation between Serum uric acid level with in hospital mortality and hospital stay.

\section{Materials and Methods}

Patients admitted with Acute ST elevation Myocardial Infarction (STEMI) in Medicine
Department for a period of three months were taken for study

\section{Inclusion Criteria}

* Patient above age of 20 and less than 75

* Admitted Within 6hr of chest pain

* Thrombolysed

\section{Exclusion Criteria}

Patient with diabetes mellitus, hypertension, metabolic syndrome, renal failure, hepatic disease, chronic alcoholics, smokers, patients on diuretics, chemotherapy, gout, malignancy were excluded.

\section{Study Design}

Patients admitted with Acute ST elevation Myocardial Infarction in medicine department. Demographic data was recorded. Histories to exclude factors that cause uric elevation were taken.BMI calculated. Clinical examination for evidence of cardiac failure or acute $\mathrm{MR}$ and $\mathrm{AR}$ was done at the time of admission. Serum Uric acid levels at the time of admission was estimated using dry chemistry method; echocardiography was done on all subjects to look for systolic and diastolic dysfunction and other mechanical complications. Any incidence of arrhythmias was noted. Duration of ICU stay and in hospital mortality was recorded. Statistical analysis is done using SPSS version 21. Biochemical and physical variables were expressed as MEAN \pm SD. Students t- test and chi square test were used to compare categorical variables. A $p$ value of $<0.01$ is considered significant.

\section{Results}

Out of 240 acute myocardial infarction cases, 148 were males and 92 were females. Male predominance was noted.

Table 1: Sex wise distribution of study group

\begin{tabular}{|l|c|c|}
\hline SEX & Number of cases & Percent \\
\hline MALE & 148 & 62 \\
\hline FEMALE & 92 & 38 \\
\hline
\end{tabular}


Fig 1: Sex wise distribution of study group

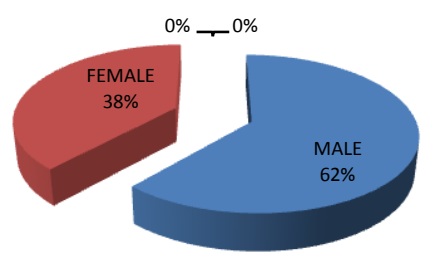

Serum uric acid level of $6 \mathrm{mg} / \mathrm{dl}$ to $7 \mathrm{mg} / \mathrm{dl}$ was noted in $53 \%$ of patients. Mean $\pm \mathrm{SD}$ of Serum uric acid level was calculated as $6.4 \pm 0.8 \mathrm{mg} / \mathrm{dl}$.

Table 2: Percentage of distribution of sample according to uric acid levels

\begin{tabular}{|l|c|c|}
\hline Uric acid & No of cases & Percent \\
\hline$<5$ & 2 & 0.8 \\
\hline $5-6$ & 80 & 33.6 \\
\hline $6-7$ & 128 & 53.3 \\
\hline$>7$ & 30 & 12.5 \\
\hline Mean \pm SD & $6.4 \pm 0.8$ & \\
\hline
\end{tabular}

Fig 2 Percentage of distribution of sample according to uric acid levels

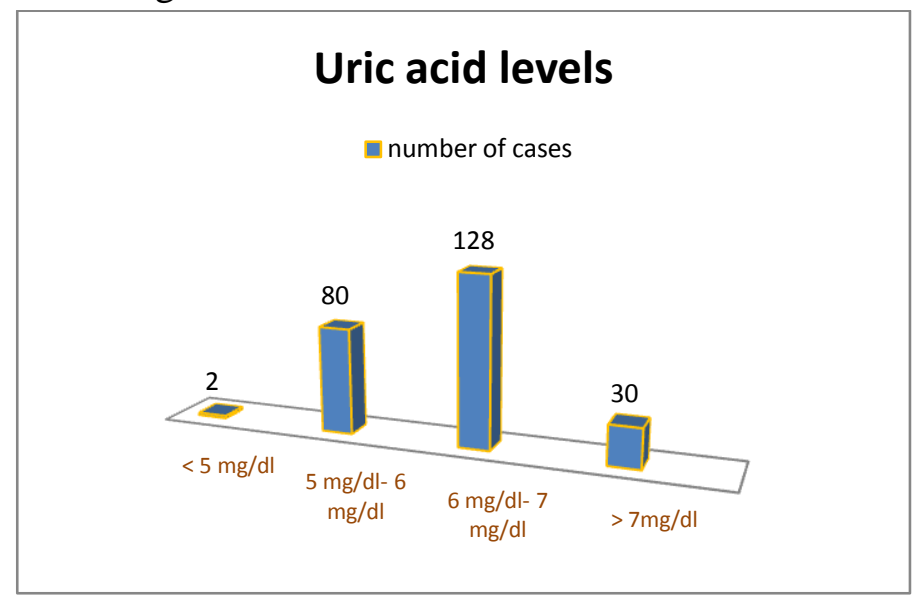

Mean \pm SD of ejection fraction was found as $50.6 \pm 7.2 \%$. $43 \%$ of patients had ejection fraction $<50$.

Table 3: Percentage of sample size according to ejection fraction

\begin{tabular}{|l|c|c|}
\hline Ejection fraction & No of cases & Percentage \\
\hline$<50$ & 104 & 43.3 \\
\hline $50-59$ & 98 & 40.8 \\
\hline$>60$ & 38 & 15.8 \\
\hline Mean \pm SD & $50.6 \pm 7.2$ & \\
\hline
\end{tabular}

Fig 3 Percentage of sample size according to ejection fraction

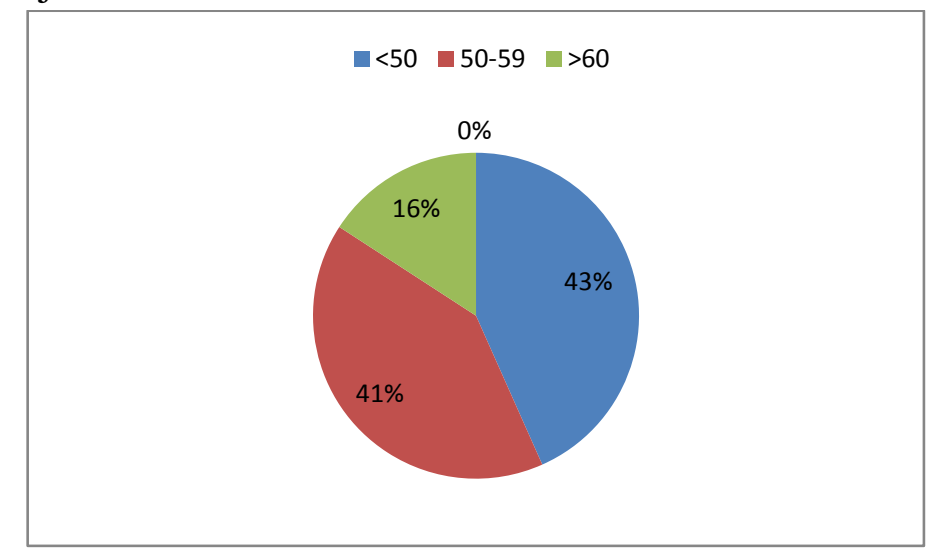

Majority of patients at the time of admission were on KILLIPS class I. (75\%)

Table 4: Percentage of distribution of the sample according to Killips class

\begin{tabular}{|l|c|c|}
\hline Killip class & Count & Percentage \\
\hline I & 180 & 75 \\
\hline II & 32 & 13.3 \\
\hline III & 16 & 6.7 \\
\hline IV & 12 & 5 \\
\hline
\end{tabular}

Fig 4 : Percentage of distribution of the sample according to Killips class

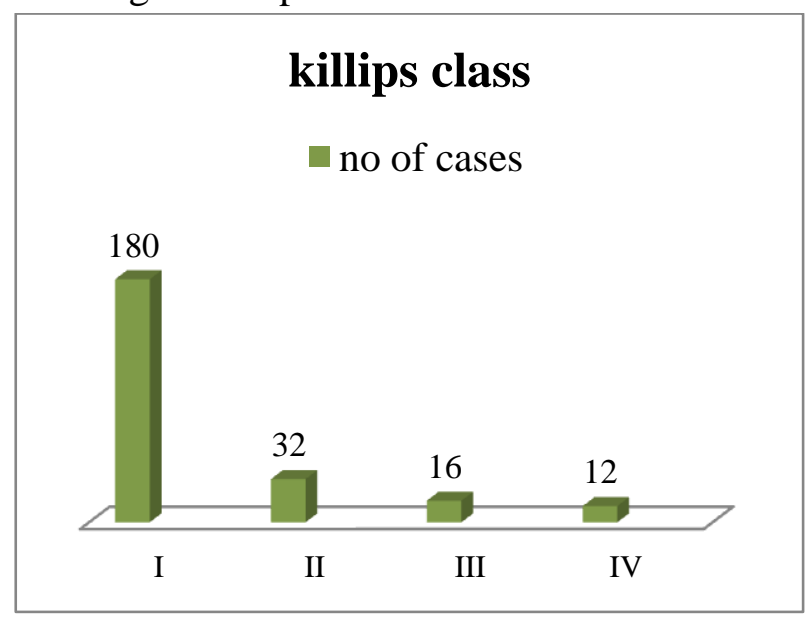

Table 5: Percentage distribution of sample according to outcome

\begin{tabular}{|l|c|c|}
\hline Outcome & Count & Percent \\
\hline Expired & 20 & 8 \\
\hline Not expired & 220 & 92 \\
\hline
\end{tabular}


Fig 5: Percentage distribution of sample according to outcome

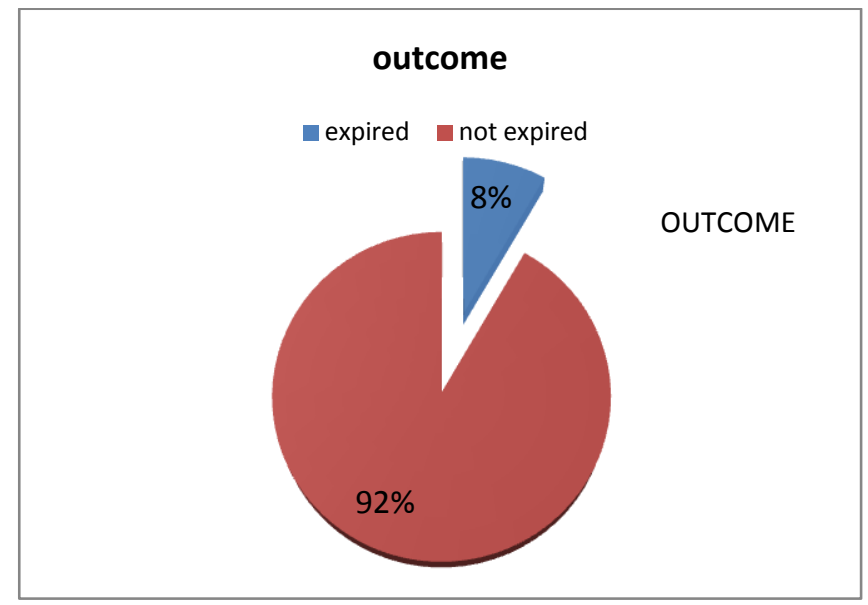

Table 6: Distribution of sample according to mechanical complication

\begin{tabular}{|l|c|c|}
\hline Complication & detected & Not detected \\
\hline MR & 30 & 210 \\
\hline AR & 12 & 228 \\
\hline TR & 10 & 230 \\
\hline VSD & 0 & 240 \\
\hline Pericardial effusion & 13 & 227 \\
\hline
\end{tabular}

Fig 6: Distribution of sample according to mechanical complication

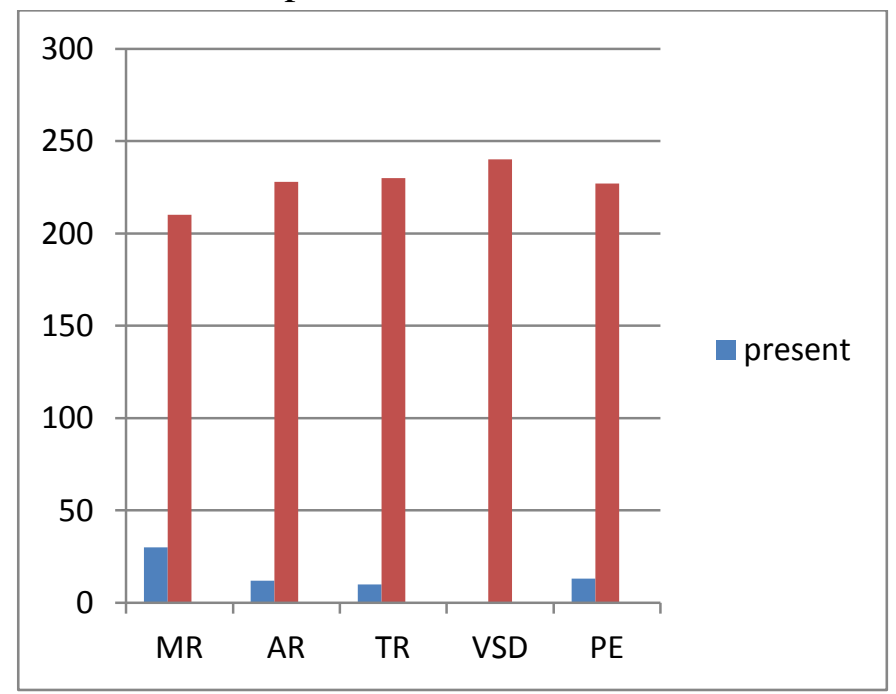

Table 7: Distribution of uric acid according to Killips class

\begin{tabular}{|l|c|c|c|c|}
\hline Uric acid & Class I & Class II & Class III & Class IV \\
\hline $5-6$ & 66 & 16 & 0 & 0 \\
\hline $6-7$ & 58 & 40 & 28 & 2 \\
\hline$>7$ & 0 & 0 & 10 & 20 \\
\hline $\mathrm{X}^{2}=93.08$ & $\mathrm{p}=0.001$
\end{tabular}

All patients with Killips class- IV had uric acid level $>7 \mathrm{mg} / \mathrm{dl}$. Patients with ejection fraction $<50$ $\%$ had mean uric acid level $6.6 \pm 0.6 \mathrm{mg} / \mathrm{dl}$.
Table 8: Comparison of uric acid based on ejection fraction levels.

\begin{tabular}{|l|c|c|}
\hline Ejection fraction & uric acid $($ Mean \pm SD) & No of cases \\
\hline$<50$ & $6.6 \pm 0.6$ & 104 \\
\hline $50-59$ & $6.3 \pm 0.22$ & 98 \\
\hline$>60$ & $6.3 \pm 0.5$ & 38 \\
\hline
\end{tabular}

$\mathrm{P}=0.039, \mathrm{f}=3.33$

Table 9: Comparison of uric acid with outcome

\begin{tabular}{|l|c|c|c|}
\hline Outcome & No of cases & Mean \pm SD & $t$ \\
\hline Expired & 12 & $7.5 \pm 0.6$ & \multirow{2}{*}{4.77} \\
Not expired & 228 & $6.4 \pm 0.6$ & \\
\hline <7hospital stay & 58 & $6.4 \pm 0.7$ & \multirow{2}{*}{0.11} \\
>7hospital stay & 182 & $6.4 \pm 0.6$ & \\
\hline ICU stay <24hr & 174 & $6.3 \pm 0.5$ & \multirow{2}{*}{4.66} \\
ICU stay>24hr & 66 & $6.8 \pm 0.7$ & \\
\hline
\end{tabular}

ICU stay, hospital stay and mortality here high for patients with elevated serum uric acid levels with a $\mathrm{p}$ value $<0.001$.

Table 10: Comparison of uric acid with Mechanical Complication

\begin{tabular}{|l|c|c|c|}
\hline Complication & No of cases & Mean \pm SD & $\mathrm{t}$ \\
\hline MR & 30 & $6.9 \pm 0.8$ & 4.39 \\
\hline AR & 13 & $7 \pm 1$ & 2.88 \\
\hline TR & 10 & $7.1 \pm 0.8$ & 3.99 \\
\hline $\begin{array}{l}\text { PERICARDIAL } \\
\text { EFFUSION }\end{array}$ & 13 & $6.4 \pm 0.08$ & 1.77 \\
\hline
\end{tabular}

Mechanical complication were more in higher uric acid level, $\mathrm{p}$ <.001.relation of uric acid with pericardial effusion was statistically insignificant.

\section{Discussion}

Study was conducted in 240 thrombolysed patients with ST ELEVATION acute myocardial infarction, admitted within 48 hours of chest pain. Of these 148 were males(61.7\%)and 92 were females(38.3\%).Uric acid was treated as a continuous variable and categorical variable. $12.5 \%$ had a uric acid level greater than $7 \mathrm{mg} / \mathrm{dl} . \mathrm{In}$ our study $53.3 \%$ had a value between $6.1-7 \mathrm{mg} / \mathrm{dl}$. Mean uric acid level was $6.4 \pm 0.6 \mathrm{mg} / \mathrm{dl}$. There was no significant difference in uric acidin males and females, contradictory to finding of Larson et $\mathrm{al}^{10}$. Patients who expired had a significantly high uric acid $7.5 \pm 0.6 \mathrm{mg} / \mathrm{dl}$. Serum uric acid level correlated with severity of cardiac failure and Killips class similar to study by Kojima Set al $^{7}$.All patients with Killips class-IV had uric acid level more than $7 \mathrm{mg} / \mathrm{dl}$, similar to study by Sinisa Car 
et $\mathrm{al}^{11}$. Serum uric acid level was inversely related to ejection fraction, mechanical complication and hospital stay. All patients who died had a uric acid level more than $6.9 \mathrm{mg} / \mathrm{dl}$ with a mean value $7.48 \mathrm{mg} / \mathrm{dl}$, serum uric acid make a significant contribution to serum anti-oxidant capacity leading to vascular injury. Treatment with allopurinol in cardiac failure might restore the endothelial function, due to anti- oxidant capacity. Study of direct effect of uric acid on vascular function was hampered by poor solubility. Newer direct study on action of uric acid on endothelial function, platelet aggregation, vessel wall elasticity and autonomic regulation of cardiovascular system required.

\section{Ethical Issues}

No ethical issues or financial grants involved in this study.

\section{Bibliography}

1. GertlerM M, Garn S.M et al.Serum uric acid in relation to age and physique in health and coronary heart disease. Ann Intern Med. 1951; 34;1421-31.

2. BengtssonC,Lapidus $\mathrm{L}$ et al. Hyperuricemia and risk of cardiovascular disease and overall death 12 yr follow up of participant in the population study of women in Gothenburg Sweden. ActaMed Scand.1988;224;549-55.

3. Bang J, Alderman et al.serum uric acid and cardiovascular mortality the NHANES 1 epidemologic follow up study 197192.JAMA 2000;283; 2404-10.

4. Ha Ieggenet al. the impact of serum uric acid in cardiovascular mortality -Life study. Kidney International.2004 65;10419.

5. Alderman M,Aiyer KJ et al. cardiovascular disease and effect of losartan.Current Med Res Opin2004;20;369-79.

6. LevyaF, Patel et al.Serum uric acid as an index of impaired oxidative metabolism in chronic cardiac failure.Arch Med Sci.2008 4(3);219-225.

7. Kojima $\mathrm{S}$ Sakamoto et al.Prognostic usefulness of serum uric acid in myocardial infarction.Am J Cardioi2005 ;96;489-90

8. Wasserman A, Shnell M et al. prognostic significance of serum uric acid in patients admitted to department of medicine.Am $\mathbf{J}$ Med Sci.2010;Jan339(1) 15-21

9. Gerber Yariv, Tanne et al serum uric acid and long term mortality from stroke and cardiovascular diease European journ of cardiovascular prevention and rehabitation 2006 volume 13 issue 2 193-98.

10. Larson et al. serum uric acid and risk of cardiovascular disease and death, The Framingham heart study.

11. SinisaCar et al. Higher uric acid on admission associated with short term mortality after MI. Current Med Jour .2009,56,559-66. 